\title{
ON VARIOUS TYPES OF VISCOUS TWO-FLUID FLOWS
}

\author{
JÜRGEN SOCOLOWSKY \\ Engineering Department - Mathematics Group, University of Applied Sciences \\ P.O. Box 2132, D-14737 Brandenburg/Havel, Germany \\ E-mail: socolowsky@fh-brandenburg.de
}

\begin{abstract}
Viscous two-fluid flows arise in different kinds of coating technologies. Frequently, the corresponding mathematical models represent two-dimensional free boundary value problems for the Navier-Stokes equations or their modifications. In this review article we present some results about nonisothermal stationary as well as about isothermal evolutionary viscous flow problems. The temperature-depending problems are characterized by coupled heat- and mass transfer and also by thermocapillary convection. The solvability of two related problems is discussed. Also, an evolutionary problem on the viscous (isothermal) flow of two connected fluids down an inclined plane is investigated.
\end{abstract}

1. Introduction and problem formulation. In this contribution we are going to give a short review about three different kinds of viscous incompressible two-fluid flows. These flows frequently describe real-world coating processes or flow regimes on parts of coating devices. In isothermal case the corresponding flows are governed by the wellknown Navier-Stokes equations. Due to the appearance of at least one free boundary (free interface and/or free surface) the associated mathematical models represent free boundary value problems. In [14] a detailed and comprehensive survey of nonstationary free boundary problems of equations of motion of both incompressible and compressible viscous fluids for the last twenty years is given.

In temperature-depending flows we include thermocapillary convection, i.e. a fluid motion driven by surface-tension gradients on a liquid-liquid interface, where these gradients arise from surface-temperature gradients and the temperature dependence of surface tension. Interesting examples of such flows may be found in the field of materials processing, particularly in coating and solidification processes or in crystal-growth processes (cf. [8]).

2000 Mathematics Subject Classification: Primary 35Q30; Secondary 35R35, 76D03.

Key words and phrases: free boundary value problems, viscous nonisothermal channel flows, Navier-Stokes equations, two-fluid flows, evolutionary flows, inclined plane.

The paper is in final form and no version of it will be published elsewhere. 
First we consider two problems for plane stationary flows with two viscous incompressible heat-conducting fluids in each (having kinematic viscosities $\nu_{i}>0$, densities $\varrho_{i}>0$ and thermal conductivities $\left.\lambda_{i}, i=1,2\right)$ through different horizontal channels. Note that the associated problems will be formulated in dimensionless form.

Let us analyze the first problem which we will denote by Problem (I) in the sequel. We investigate the two-fluid flow within a perturbed horizontal channel of width 1 between the walls $S_{0}$ and $S_{2}$ (cf. Fig. 1). The moving bottom $S_{0}$ of it is given by the formula $S_{0}=\left\{\mathbf{x}=\left(x_{1}, x_{2}\right) \in \mathbb{R}^{2}: x_{2}=0,-\infty<x_{1}<+\infty\right\}$ and the fixed top $S_{2}$ has the representation $S_{2}=\left\{\mathbf{x} \in \mathbb{R}^{2}: x_{2}=1+\varepsilon \varphi_{2}\left(x_{1}\right),-\infty<x_{1}<+\infty\right\}$. Furthermore, we suppose that $\varphi_{2}$ has a compact support. Since the channel is horizontal, the direction $\mathbf{e}_{g}$ of the gravitational force is equal to $(0,-1)^{T}$ (cf. Fig. 1).

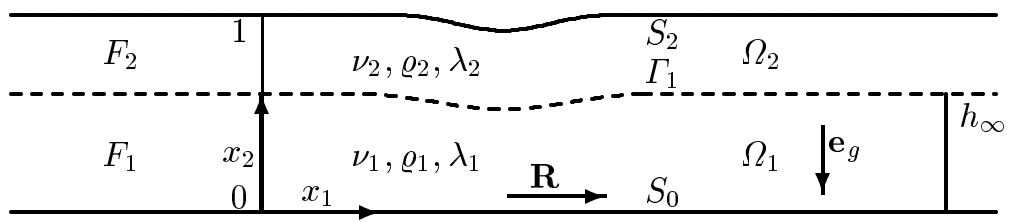

Fig. 1. Flow domain for Problem (I)

We study the plane stationary flow of two viscous incompressible heat-conducting fluids generated by a pressure gradient downstream in the perturbed channel, by a temperature gradient in the transverse direction and by motion of the lower wall $S_{0}$ with constant velocity $\mathbf{R}=(R, 0)^{T}$. This means mathematically that the volume flux $F_{i}$ in each fluid layer $\Omega_{i}(i=1,2)$ is prescribed. Suppose that the free interface $\Gamma_{1}$ separating two fluid layers admits the parametrization $\Gamma_{1}=\left\{\mathbf{x} \in \mathbb{R}^{2}: x_{2}=\psi_{1}\left(x_{1}\right),-\infty<x_{1}<+\infty\right\}$, where the function $\psi_{1}$ is a priori unknown and has to be found. Emphasize that $F_{i}(i=1,2), R$ are not necessarily positive for this channel flow.

Let $h_{\infty}$ be the constant limit of $\psi_{1}\left(x_{1}\right)$ at both infinities. Obviously, we should have $0<h_{\infty}<1$. Problem (I) has the following form: find a vector of velocity $\mathbf{v}=$ $\left(v_{1}\left(x_{1}, x_{2}\right), v_{2}\left(x_{1}, x_{2}\right)\right)^{T}$, a pressure $p\left(x_{1}, x_{2}\right)$, a temperature $\theta\left(x_{1}, x_{2}\right)$ and a function $\psi_{1}\left(x_{1}\right)$ satisfying in the domain $\Omega=\Omega_{1} \cup \Omega_{2}$ with $\Omega_{1}=\left\{\mathbf{x} \in \mathbb{R}^{2}: 0<x_{2}<\right.$ $\left.\psi_{1}\left(x_{1}\right),-\infty<x_{1}<+\infty\right\}$ and $\Omega_{2}=\left\{\mathbf{x} \in \mathbb{R}^{2}: \psi_{1}\left(x_{1}\right)<x_{2}<1+\varepsilon \varphi_{2}\left(x_{1}\right),-\infty<\right.$ $\left.x_{1}<+\infty\right\}$ the Boussinesq-approximation of the coupled heat- and mass transfer (cf. [2])

$$
\left\{\begin{array}{l}
(\mathbf{v} \cdot \nabla) \mathbf{v}-\nu \nabla^{2} \mathbf{v}+\frac{1}{\varrho} \nabla p=(g-\gamma \theta) \mathbf{e}_{g}, \\
\nabla \cdot \mathbf{v}=0, \\
(\mathbf{v} \cdot \nabla) \theta-\lambda \nabla^{2} \theta=0
\end{array}\right.
$$

and the boundary and integral conditions

$$
\begin{array}{cr}
\left.\mathbf{v}\right|_{S_{0}}=(R, 0)^{T}, & \left.\mathbf{v}\right|_{S_{2}}=\mathbf{0}, \\
\left.\theta\right|_{S_{0}}=\theta_{0}, & \left.\theta\right|_{S_{2}}=\theta_{2},
\end{array}
$$




$$
\left\{\begin{array}{c}
{\left.[\theta]\right|_{\Gamma_{1}}=0,\left.\quad\left[\lambda \frac{\partial \theta}{\partial n}\right]\right|_{\Gamma_{1}}=0,\left.\quad[\mathbf{v}]\right|_{\Gamma_{1}}=\mathbf{0},} \\
\left.\mathbf{v} \cdot \mathbf{n}\right|_{\Gamma_{1}^{-}}=0,\left.\quad[\mathbf{t} \cdot \mathbf{S}(\mathbf{v}) \mathbf{n}]\right|_{\Gamma_{1}}=-\left.b \frac{\partial \theta}{\partial t}\right|_{\Gamma_{1}^{-}}, \\
\frac{\mathrm{d}}{\mathrm{d} x_{1}} \frac{\psi_{1}^{\prime}\left(x_{1}\right)}{\sqrt{1+\psi_{1}^{\prime}\left(x_{1}\right)^{2}}}=\left.\frac{1}{\sigma(\theta)}[-p+\mathbf{n} \cdot \mathbf{S}(\mathbf{v}) \mathbf{n}]\right|_{\Gamma_{1}}, \\
\lim _{\left|x_{1}\right| \rightarrow+\infty} \psi_{1}\left(x_{1}\right)=h_{\infty}, \quad \int_{\delta_{1}(\widehat{q})} v_{1} \mathrm{~d} x_{2}=F_{1}, \\
\int_{\delta_{2}(\widehat{q})} v_{1}\left(\widehat{q}, x_{2}\right) \mathrm{d} x_{2}=F_{2} .
\end{array}\right.
$$

In [8] it was shown that for a large number of liquids the surface tension $\sigma$ can be regarded as a linear function of the temperature $\theta$ along the free interface $\Gamma_{1}$

$$
\sigma(\theta)=a-b \theta \quad(a, b>0) .
$$

In Problem (I) the symbol $\delta_{i}(\widehat{q})$ denotes the intersection of $\Omega_{i}$ with the vertical line $x_{1}=\widehat{q}$. By $\gamma_{m}$ we denote the thermal expansion coefficient of the $m$-th fluid $(m=1,2)$. The symbol $g$ means the acceleration of gravity. The values $\theta_{0}$ and $\theta_{2}$ are the (constant) given temperatures of the walls $S_{0}$ and $S_{2}$, respectively. Without loss of generality one can suppose that $\theta_{0}=0$ and that $\theta$ is in fact the difference between the physical temperature and $\theta_{0}$.

Furthermore, the following notations have been used: $\mathbf{n}$ and $\mathbf{t}$ are unit vectors normal and tangential to $\Gamma_{1}$ and oriented as $x_{2}, x_{1}$, respectively. By $\mathbf{a} \cdot \mathbf{b}$ we mean the inner product of $\mathbf{a}, \mathbf{b} \in \mathbb{R}^{2}, \nabla=\left(\partial / \partial x_{1}, \partial / \partial x_{2}\right)^{T}$ is the gradient operator, $\nabla p=\operatorname{grad} p, \nabla \cdot \mathbf{v}=$ $\operatorname{div} \mathbf{v},\left.\varrho\right|_{\Omega_{m}}=\varrho_{m}(m=1,2)$ is the restriction of $\varrho$ to $\Omega_{m}$ (analogously for $\nu$ and $\left.\lambda\right)$. $\nabla^{2}$ denotes the Laplace operator. By $\mathbf{S}(\mathbf{v})$ we denote the deviatoric stress tensor, i.e. the matrix with elements $S_{i j}(\mathbf{v})=\varrho \nu\left(\partial v_{i} / \partial x_{j}+\partial v_{j} / \partial x_{i}\right)(i, j=1,2)$. The symbol $\left.[w]\right|_{\Gamma_{1}}$ expresses the jump of $w$ crossing the free interface $\Gamma_{1}$, i.e.

$$
\left.\left[w\left(\mathbf{x}_{0}\right)\right]\right|_{\Gamma_{1}}:=\lim _{\mathbf{y} \rightarrow \mathbf{x}_{0}} w(\mathbf{y})-\lim _{\mathbf{x} \rightarrow \mathbf{x}_{0}} w(\mathbf{x}), \quad\left(\mathbf{x}_{0} \in \Gamma_{1}, \mathbf{y} \in \Omega_{1}, \mathbf{x} \in \Omega_{2}\right),
$$

and the symbol $\left.w\right|_{\Gamma_{1}^{-}}$denotes the limit from below at the interface $\Gamma_{1}$, more precisely

$$
\left.w\left(\mathbf{x}_{0}\right)\right|_{\Gamma_{1}^{-}}:=\lim _{\mathbf{y} \rightarrow \mathbf{x}_{0}} w(\mathbf{y}), \quad\left(\mathbf{x}_{0} \in \Gamma_{1}, \mathbf{y} \in \Omega_{1}\right) .
$$

Note that the left-hand side of $(4)_{6}$ (i.e. of the sixth equation in (4)) is equal to the curvature $K\left(x_{1}\right)$ of $\Gamma_{1}$. Throughout this paper we denote by $r$ the rheological ratio $r:=$ $\nu_{1} \varrho_{1} /\left(\nu_{2} \varrho_{2}\right)$.

The second flow under consideration is also steady-state and has some features of a slot coating process. The channel is again horizontal, unbounded in both directions and contains a semi-infinite inner wall (cf. Fig. 2). The lower wall $S_{0}:=\left\{\mathbf{x} \in \mathbb{R}^{2}:-\infty<x_{1}<\right.$ $\left.+\infty, x_{2}=0\right\}$ is again moving with constant velocity $\mathbf{R}=(R, 0)^{T}(R \geq 0)$. The upper wall (which is a straight line in this case) $S_{2}:=\left\{\mathbf{x} \in \mathbb{R}^{2}:-\infty<x_{1}<+\infty, x_{2}=1\right\}$ is at rest. Furthermore, we are given the partial inner wall $S_{3}:=\left\{\mathbf{x} \in \mathbb{R}^{2}:-\infty<x_{1}<0, x_{2}=\right.$ $\left.h_{1}\left(0<h_{1}<1\right)\right\}$. Thus, in fact we have two separated parallel channels for negative values of $x_{1}$. Both viscous fluids flow out of the two channels and behind the point $Q\left(0, h_{1}\right)$ they join and create a free interface $\Gamma_{1}:=\left\{\mathbf{x} \in \mathbb{R}^{2}: 0<x_{1}<+\infty, x_{2}=\psi_{1}\left(x_{1}\right)\right\}$ where $\psi_{1}$ is 
unknown a priori and has to be found. It is supposed that the free interface $\Gamma_{1}$ separates from the inner wall $S_{3}$ at its endpoint $Q$.

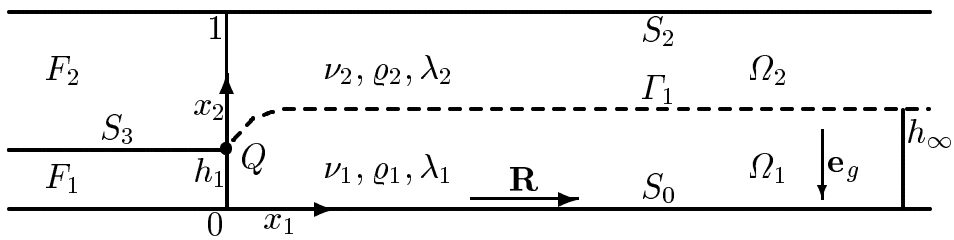

Fig. 2. Flow domain for Problem (II)

By $\Omega_{1}:=\left\{\mathbf{x} \in \mathbb{R}^{2}: 0<x_{2}<h_{1}\right.$ if $-\infty<x_{1} \leq 0$ and $0<x_{2}<\psi_{1}\left(x_{1}\right)$ if $0<x_{1}<$ $+\infty\}$ we denote the flow domain of the lower fluid. The flow domain of the upper fluid is $\Omega_{2}:=\left\{\mathbf{x} \in \mathbb{R}^{2}: h_{1}<x_{2}<1\right.$ if $-\infty<x_{1} \leq 0$ and $\psi_{1}\left(x_{1}\right)<x_{2}<1$ if $\left.0<x_{1}<+\infty\right\}$. Finally, $\Omega:=\Omega_{1} \cup \Omega_{2}$ is the union of both fluid layers. The direction of the gravitational force is again the vector $\mathbf{e}_{g}=(0,-1)^{T}$. We study the two-fluid flow through the channel $\Omega$ caused by a pressure gradient downstream, by temperature gradients in the spanwise direction and by motion of the lower channel wall. This means mathematically that the positive volume flux $F_{i}$ in each liquid layer $\Omega_{i}(i=1,2)$ is prescribed and the final fluid layer thicknesses $h_{\infty}$ and $\left(1-h_{\infty}\right)$ are to be determined.

An interpretation of such a flow could be the flow of two liquids coming from different reservoirs (i.e. slots or chambers) and flowing together in one channel after their unification. In slot coaters such flows occur on some parts of the coater. The corresponding motion as well as the final layer thicknesses are important in that case.

Let $h_{\infty}$ be the constant limit of $\psi_{1}\left(x_{1}\right)$ as $x_{1} \rightarrow+\infty$. Obviously, $0<h_{\infty}<1$. Then Problem (II) has the following description: to find a vector of velocity $\mathbf{v}$, a pressure $p$, a temperature $\theta$ and a function $\psi_{1}$ satisfying in the domain $\Omega$ the Boussinesqapproximation of the coupled heat- and mass transfer

$$
\left\{\begin{array}{l}
(\mathbf{v} \cdot \nabla) \mathbf{v}-\nu \nabla^{2} \mathbf{v}+\frac{1}{\varrho} \nabla p=(g-\gamma \theta) \mathbf{e}_{g} \\
\nabla \cdot \mathbf{v}=0 \\
(\mathbf{v} \cdot \nabla) \theta-\lambda \nabla^{2} \theta=0
\end{array}\right.
$$

and the boundary and integral conditions

$$
\begin{gathered}
\left.\mathbf{v}\right|_{S_{0}}=(R, 0)^{T},\left.\quad \mathbf{v}\right|_{S_{2}}=\mathbf{0},\left.\quad \mathbf{v}\right|_{S_{3}^{ \pm}}=\mathbf{0}, \\
\left.\theta\right|_{S_{0}}=\theta_{0},\left.\quad \theta\right|_{S_{2}}=\theta_{2},\left.\quad \theta\right|_{S_{3}^{ \pm}}=\theta_{3}, \\
\left\{\begin{array}{c}
{\left.[\theta]\right|_{\Gamma_{1}}=0,\left.\quad\left[\lambda \frac{\partial \theta}{\partial n}\right]\right|_{\Gamma_{1}}=0,\left.\quad[\mathbf{v}]\right|_{\Gamma_{1}}=\mathbf{0},} \\
\left.\mathbf{v} \cdot \mathbf{n}\right|_{\Gamma_{1}^{-}}=0,\left.\quad[\mathbf{t} \cdot \mathbf{S}(\mathbf{v}) \mathbf{n}]\right|_{\Gamma_{1}}=-\left.b \frac{\partial \theta}{\partial t}\right|_{\Gamma_{1}^{-}}, \\
\frac{\mathrm{d}}{\mathrm{d} x_{1}} \frac{\psi_{1}^{\prime}\left(x_{1}\right)}{\sqrt{1+\psi_{1}^{\prime}\left(x_{1}\right)^{2}}}=\left.\frac{1}{\sigma(\theta)}[-p+\mathbf{n} \cdot \mathbf{S}(\mathbf{v}) \mathbf{n}]\right|_{\Gamma_{1}}, \\
\lim _{x_{1} \rightarrow+\infty} \psi_{1}\left(x_{1}\right)=h_{\infty}, \quad \int_{\delta_{1}(\widehat{q})} v_{1} \mathrm{~d} x_{2}=F_{1},
\end{array}\right.
\end{gathered}
$$




$$
\int_{\delta_{2}(\widehat{q})} v_{1}\left(\widehat{q}, x_{2}\right) \mathrm{d} x_{2}=F_{2} .
$$

Note that surface tension is the same as in (6). For the one-side limits at $S_{3}^{ \pm}$we use analogous symbols as in (8). The fluid layer thickness $h_{\infty}$ has to be determined.

Problem (III) models a nonstationary two-fluid free surface flow down an inclined plane (cf. Fig. 3). The flow is isothermal, two-dimensional and both fluids are incompressible and viscous. The motion is generated only by gravity in this case since the gravitational force is directed along the vector $\mathbf{e}_{g}=(\sin \alpha,-\cos \alpha)^{T}$ where $\alpha$ with $0<\alpha<\pi / 2$ is the inclination angle of the plane (line) $S$. The uniform layer heights $h_{1}, h_{2}\left(h_{2}>h_{1}>0\right)$ that are approximated at both infinities are prescribed in this problem. In the literature (see e.g. [14]) similar problems are called viscous surface wave problems. In the described free boundary value problem one has to find the flow domain $\Omega_{t}=\Omega_{t}^{(1)} \cup \Omega_{t}^{(2)}(t \geq 0)$ occupied by two fluids and therein the flow fields $v_{1}\left(t, x_{1}, x_{2}\right), v_{2}\left(t, x_{1}, x_{2}\right), p\left(t, x_{1}, x_{2}\right)$ for velocity components and pressure. The (a priori unknown) flow domain is described by the functions $x_{2}=\psi_{j}\left(t, x_{1}\right)(j=1,2)$ characterizing the free interface (or surface, resp.) $\Gamma_{t}^{(j)}$. We emphasize that the initial flow domain, i.e. the domain $\Omega_{t}$ at $t=0$ is given by $\Omega_{0}$ together with the initial positions of the free boundaries $\Gamma_{0}^{(j)}(j=1,2)$ which are prescribed by the functions $x_{2}=\psi_{j}\left(0, x_{1}\right)=\psi_{j}^{(0)}\left(x_{1}\right)$. Let us remark that in the sequel $\mathbf{t}=\left(t_{1}, t_{2}\right)^{T}$ denotes again the unit tangential vector along the free boundaries whereas $t$ denotes the time.

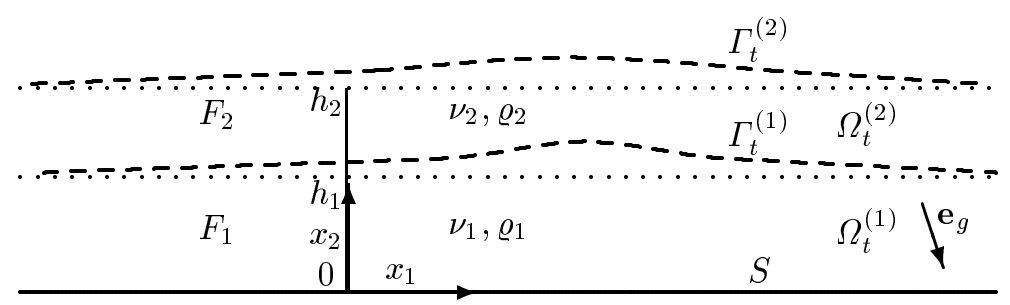

Fig. 3. Flow domain for Problem (III)

For $\mathbf{x} \in \Omega_{t}$ and $t \in(0, T)$ one obtains the well-known time-dependent Navier-Stokes equations

$$
\left\{\begin{array}{l}
\partial_{t} \mathbf{v}+\varrho(\mathbf{v} \cdot \nabla) \mathbf{v}-\nu \varrho \nabla^{2} \mathbf{v}+\nabla p=\varrho g \mathbf{e}_{g} \\
\nabla \cdot \mathbf{v}=0
\end{array}\right.
$$

together with the initial condition and the no-slip boundary condition

$$
\left.\mathbf{v}\right|_{t=0}=\mathbf{v}_{0}, \quad\left(x \in \Omega_{0}\right),\left.\quad \mathbf{v}\right|_{S}=\mathbf{0}, \quad(t \in(0, T)),
$$

the boundary conditions on the free interface

$$
\left\{\begin{array}{l}
{\left.[\mathbf{v}]\right|_{\Gamma_{t}^{(1)}}=\mathbf{0},\left.\quad \mathbf{v} \cdot \mathbf{n}\right|_{\Gamma_{t}^{(1),-}}=\frac{\partial_{t} \psi_{1}}{\sqrt{1+\left|\partial_{1} \psi_{1}\right|^{2}}},} \\
{\left.[\mathbf{t} \cdot \mathbf{S}(\mathbf{v}) \mathbf{n}]\right|_{\Gamma_{t}^{(1)}}=0,\left.\quad \frac{1}{\sigma_{1}}[-p+\mathbf{n} \cdot \mathbf{S}(\mathbf{v}) \mathbf{n}]\right|_{\Gamma_{t}^{(1)}}=\partial_{1} \frac{\partial_{1} \psi_{1}}{\sqrt{1+\left|\partial_{1} \psi_{1}\right|^{2}}},}
\end{array}\right.
$$


and the boundary conditions on the free surface

$$
\left\{\begin{array}{l}
\left.\left.\mathbf{v} \cdot \mathbf{n}\right|_{\Gamma_{t}^{(2)}}=\frac{\partial_{t} \psi_{1}}{\sqrt{1+\left|\partial_{1} \psi_{2}\right|^{2}}}, \quad \mathbf{t} \cdot \mathbf{S}(\mathbf{v}) \mathbf{n}\right)\left.\right|_{\Gamma_{t}^{(2)}}=0 \\
\left.\frac{1}{\sigma_{2}}\left(p_{a}-p+\mathbf{n} \cdot \mathbf{S}(\mathbf{v}) \mathbf{n}\right)\right|_{\Gamma_{t}^{(2)}}=\partial_{1} \frac{\partial_{1} \psi_{2}}{\sqrt{1+\left|\partial_{1} \psi_{2}\right|^{2}}} .
\end{array}\right.
$$

In Problem (III) the notations $\partial_{j}:=\partial / \partial x_{j}(j=1,2), \partial_{t}:=\partial / \partial t, \Omega_{0}=\left.\Omega_{t}\right|_{t=0}$ have additionally been used. Here the surface tensions $\sigma_{j}(j=1,2)$ are constant. In Eq. (17) the value $p_{a}$ denotes the given (atmospheric) pressure outside the flow domain.

2. General solution schemes. There is an extensive mathematical literature on problems for stationary or nonstationary flows of viscous incompressible fluids with one or more free boundaries. Numerous references on this topic can be found e.g. in the bibliographies of [4], [7]. In the analytical study [8] the temperature dependence was additionally taken into account. Coating flows which frequently include static or dynamic contact points were studied in [5], [9-12]. In all papers containing either compact or semi-infinite free boundary value problems the same general solution scheme developed first in [3] has been used. This solution technique is sometimes called normal stress iteration scheme.

Let us briefly recall this scheme for Problem (II). The original problem is divided into two problems: the boundary value problem for the differential equations (9) in a fixed domain and the problem of finding the free boundary $\Gamma_{1}$ from the equation

$$
K\left(x_{1}\right)=\left.\frac{1}{\sigma(\theta)}[-p(\mathbf{x})+\mathbf{n} \cdot \mathbf{S}(\mathbf{v}) \mathbf{n}]\right|_{\Gamma_{1}},
$$

which is called the normal stress boundary condition, and from the associated boundary conditions at the endpoints. The solution of the free boundary problem can be found by the method of successive approximations. At every step of successive approximations the system (9) is solved in a fixed domain. The obtained solution is substituted into the right-hand side of (18) and solving this equation implies the next iterate for the free boundary $\Gamma_{1}$. Thus, one gets a new domain in which system (9) has to be solved again. So, this scheme can be illustrated by the diagram

$$
\Gamma_{1}^{0} \rightarrow \Omega^{0} \rightarrow\left(\mathbf{v}^{1}, p^{1}, \theta^{1}\right) \rightarrow \Gamma_{1}^{1} \rightarrow \Omega^{1} \rightarrow\left(\mathbf{v}^{2}, p^{2}, \theta^{2}\right) \rightarrow \ldots
$$

But on the other hand, for free boundary problems in which the unknown flow domain is unbounded in two directions as in Problem (I) the described scheme is not applicable (cf. [4], [7] and others).

In order to solve Problem (I) a different scheme was proposed in [4], and independently in [1]. This scheme is based on a linearization of the original problem on an appropriate exact solution in the unperturbed "uniform" flow domain, say $\Pi=\left\{\mathbf{x} \in \mathbb{R}^{2}: 0<x_{2}<\right.$ $1\}$. The main difference of this scheme from the previous one is that on each step of iterations the determination of $\mathbf{v}, p, \theta$ is not separated from the determination of the free boundary $\Gamma_{1}$ (i.e. from the determination of the function $\psi_{1}$ describing $\Gamma_{1}$ ).

For Problem (I) this scheme can be illustrated by the diagram

$$
\left(\mathbf{v}^{0}, p^{0}, \theta^{0}, \psi_{1}^{0}\right) \rightarrow\left(\mathbf{v}^{1}, p^{1}, \theta^{1}, \psi_{1}^{1}\right) \rightarrow \ldots \rightarrow\left(\mathbf{v}^{m}, p^{m}, \theta^{m}, \psi_{1}^{m}\right) \rightarrow \ldots
$$


where on each step of iterations the linearized problem is solved in the same "uniform" domain and the functions $\mathbf{v}, p, \theta$ and $\psi_{i}(i=1,2)$ are determined simultaneously.

Note that Problem (III) is solved using a very similar linearization scheme compared with (20). A corresponding problem for one viscous fluid flow was investigated in [13].

3. Solvability results. We are beginning with the investigation of Problem (I). Let $\Pi_{m}(m=1,2)$ be the strip-like domains

$$
\begin{aligned}
& \Pi_{1}:=\left\{\mathbf{x} \in \mathbb{R}^{2}: 0<x_{2}<h_{\infty},-\infty<x_{1}<+\infty\right\}, \\
& \Pi_{2}:=\left\{\mathbf{x} \in \mathbb{R}^{2}: h_{\infty}<x_{2}<1,-\infty<x_{1}<+\infty\right\},
\end{aligned}
$$

and $\Pi=\Pi_{1} \cup \Pi_{2}$ their union, where $h_{\infty} \in(0,1)$ is the root to equation (21) (or is one of the roots to Eq.(21)). The weighted Sobolev spaces on $\Pi$ are introduced in detail in [7], [10]. Let us now present the main result on Problem (I).

TheOREM 1. Let $S_{2}=\left\{\mathbf{x} \in \mathbb{R}^{2}: x_{2}=1+\varepsilon \varphi_{2}\left(x_{1}\right),-\infty<x_{1}<+\infty\right\}, \varphi_{2} \in W_{\beta}^{l+5 / 2,2}(\mathbb{R})$ with $l \geq 0, \beta=\delta\left|\beta_{0}\right|>0$, where $\beta_{0}$ is independent of $\delta$ and depends on eigenvalues of the operator pencils associated with the corresponding linear problem (cf. [7]). Assume that $\delta$ is sufficiently small. Then there exist positive numbers $\widehat{\varepsilon}, \widehat{r}$ such that for every $\varepsilon \in(0, \widehat{\varepsilon})$ Problem (I) has a unique solution $\left(\mathbf{v}, p, \theta, \psi_{1}\right)^{T}$. The solution admits the representation

$$
\begin{array}{rrr}
\mathbf{v}(\mathbf{x})=\mathbf{v}^{0}(\mathbf{x})+\varepsilon \mathbf{u}(\mathbf{x}), & p(\mathbf{x})=p^{0}(\mathbf{x})+\varepsilon q(\mathbf{x}), \\
\theta(\mathbf{x})=\theta^{0}(\mathbf{x})+\varepsilon \vartheta(\mathbf{x}), & \psi_{1}\left(x_{1}\right)=h_{\infty}+\varepsilon \Psi_{1}\left(x_{1}\right),
\end{array}
$$

where $h_{\infty} \in(0,1)$ is one of the roots to equation $(21),\left\{\mathbf{v}^{0}, p^{0}, \theta^{0}\right\}$ are the functions of the basic solution in the uniform unperturbed domain $\Pi$,

$\mathbf{U}:=\left(\mathbf{u}, q, \vartheta, \Psi_{1}\right)^{T} \in\left[W_{\beta}^{l+2,2}(\Pi)\right]^{2} \times W_{\beta}^{l+1,2}(\Pi) \times W_{\beta}^{l+2,2}(\Pi) \times W_{\beta}^{l+5 / 2,2}(\mathbb{R}) \equiv \mathcal{D}_{\beta}^{l, 2} W(\Pi)$ and the following inequalities hold

$$
\left\|\mathbf{U} ; \mathcal{D}_{\beta}^{l, 2} W(\Pi)\right\| \leq \widehat{r}, \quad \widehat{\varepsilon} \leq \text { const } \cdot \delta^{2} .
$$

Note that due to the possible existence of more than one root $h_{\infty}$ to Eq.(21) there can be more than one solution to Problem (I). This kind of nonuniqueness was already studied for isothermal flows in [7]. A first example of analogous nonuniqueness was presented in [6]. Furthermore, let us remark that $\mathbf{U}=\left(\mathbf{u}, q, \vartheta, \Psi_{1}\right)^{T}$ in Theorem 1 is the unique solution of an associated linear boundary value problem that was obtained by linearization of the original Problem (I) over the basic solution $\left\{\mathbf{v}^{0}, p^{0}, \theta^{0}\right\}$ in the uniform unperturbed (strip-like) domain $\Pi$. Theorem 1 is proven in [10].

We proceed with the solvability of Problem (II). By straightforward calculations one can determine the exact nonisothermal Poiseuille flows $\left\{\mathbf{v}^{(-)}(\mathbf{x}), p^{(-)}(\mathbf{x}), \theta^{(-)}(\mathbf{x})\right\}$, $\mathbf{x} \in \Omega^{-}$in the left-hand part $\Omega^{-}$of the (double) channel. The corresponding velocities and temperatures do not depend on $x_{1}$ and they are written down in detail in [10].

By $\left\{\mathbf{v}^{0}, p^{0}, \theta^{0}\right\}$ we denote the exact solution (nonisothermal Poiseuille flow) in the united part $\Omega^{+}$at the right-hand side of the channel. Remark that this solution coincides with the basic solution to Problem (I). That solution was also determined by straightforward calculations in the Appendix of the reference [10]. The associated flow 
fields are given therein. An essential part of the determination of $\left\{\mathbf{v}^{0}, p^{0}, \theta^{0}\right\}$ consists in the calculation of the value $h_{\infty}$ from the following 5 th degree polynomial equation

$$
\begin{aligned}
& r(r-1) R h_{\infty}^{5}+\left[-4 r(r-1) R-r(r-1) F_{1}-(r-1) F_{2}\right] h_{\infty}^{4} \\
& +\left[r(6 r-5) R+2 r(2 r-3) F_{1}-2 r F_{2}\right] h_{\infty}^{3}+[2 r(-2 r+1) R \\
& \left.+3 r(-2 r+3) F_{1}+3 r F_{2}\right] h_{\infty}^{2}+\left[r^{2} R+4 r(r-1) F_{1}\right] h_{\infty}-r^{2} F_{1}=0 .
\end{aligned}
$$

Equation (21) coincides with equation (A.13) from [7] when the channel is horizontal. Note that the final thickness $h_{\infty}$ is a function of $F_{1}, F_{2}, R$ and of the rheological parameters of the fluids. It can have up to three different values in the interval $(0,1)$ for the same parameter set (cf. [7]). Furthermore, by $\psi_{1}^{0}\left(x_{1}\right)$ we denote the infinitely differentiable solution of the following boundary value problem

$$
\left\{\begin{array}{l}
\frac{\mathrm{d}}{\mathrm{d} x_{1}} \frac{\psi_{1}^{\prime}\left(x_{1}\right)}{\sqrt{1+\psi_{1}^{\prime}\left(x_{1}\right)^{2}}}-\frac{g\left(\varrho_{1}-\varrho_{2}\right)}{\sigma(0)} \psi_{1}\left(x_{1}\right)=-\frac{g\left(\varrho_{1}-\varrho_{2}\right)}{\sigma(0)} h_{\infty}, \\
\psi_{1}(0)=h_{1}, \quad \lim _{x_{1} \rightarrow+\infty} \psi_{1}\left(x_{1}\right)=h_{\infty},
\end{array}\right.
$$

which can be obtained from $(12)_{6}$ by setting $\mathbf{v}=\mathbf{0}, p=$ const., $\theta=0$ as the starting solution for $F_{1}=F_{2}=R=\theta_{0}=\theta_{2}=0$. Let $\xi=\xi\left(x_{1}\right)$ be a smooth cut-off function vanishing for $\left|x_{1}\right| \leq 1$ and equal to 1 for $\left|x_{1}\right| \geq 2$. Finally, suppose that $\varrho_{1}>\varrho_{2}$. Now we can formulate the main result about Problem (II).

THEOREM 2. There exist positive real numbers $s_{0}, M_{0}$ and $z_{0} \leq \sqrt{g\left(\varrho_{1}-\varrho_{2}\right) / \sigma(0)}$ such that for arbitrary $s \in\left(0, s_{0}\right), z \in\left(0, z_{0}\right), \max \left(F_{1}, F_{2}, R,\left|\theta_{0}\right|,\left|\theta_{2}\right|\right)<M_{0}$ and for positive $h_{\infty}, F_{1}, F_{2}, R$ satisfying the condition

$$
\left|h_{1}-h_{\infty}\left(F_{1}, F_{2}, R\right)\right|<\sqrt{\frac{2 \sigma(0)}{g\left(\varrho_{1}-\varrho_{2}\right)}},
$$

Problem (II) has a unique solution $\left\{v, p, \theta, \psi_{1}\right\}$ which can be represented in the form

$$
\begin{array}{ll}
\mathbf{v}=\xi\left(-x_{1}\right) \mathbf{v}^{(-)}+\xi\left(x_{1}\right) \mathbf{v}^{0}+\mathbf{w}, & p=\xi\left(-x_{1}\right) p^{(-)}+p^{0}+q, \\
\theta=\xi\left(-x_{1}\right) \theta^{(-)}+\xi\left(x_{1}\right) \theta^{0}+\vartheta, & \psi_{1}\left(x_{1}\right)=\psi_{1}^{0}\left(x_{1}\right)+\omega\left(x_{1}\right),
\end{array}
$$

where $\left\{\mathbf{v}^{(-)}, p^{(-)}, \theta^{(-)}\right\}$denotes the nonisothermal Poiseuille flow in both channels as $x_{1} \rightarrow-\infty$ and $\left\{\mathbf{v}^{0}, p^{0}, \theta^{0}\right\}$ is the basic solution of Problem (I) as $x_{1} \rightarrow+\infty$. The function $\xi$ denotes the smooth cut-off function mentioned above. Moreover, $\mathbf{w} \in\left[C_{s, z}^{s+2}(\Omega)\right]^{2}, \vartheta \in$ $C_{s, z}^{s+2}(\Omega), q \in C_{s-1, z}^{s+1}\left(\Omega^{0} \cup \Omega^{+}\right), \nabla q \in C_{s-2, z}^{s}(\Omega)$ and $\omega \in C_{1+s, z}^{3+s}\left(\mathbb{R}_{+}^{1}\right)$ hold.

The proof of this theorem and also the precise definition of weighted Hölder spaces can be found in [10]. The condition (23) is a consequence of solving the boundary value problem $(22)$ and the restriction $\varrho_{1}>\varrho_{2}$ is essential for the applied method. The weight parameter $s_{0}$ in Theorem 2 can be estimated investigating a model problem for a nonisothermal Stokes system in a neighborhood of $Q$ in the same way as in [9]. The exponential decay of $\{\mathbf{w}, q, \vartheta, \omega\}$ at infinity is well-known (cf. [4], [9]).

Finally, we are going to analyze Problem (III). A similar nonstationary roblem for an one fluid free surface flow was already treated in [13] (see also [14]). Again, let $\Pi$ be the uniform (unperturbed) domain $\Pi:=\left\{\mathbf{x}=\left(x_{1}, x_{2}\right) \in \mathbb{R}^{2}, 0<x_{2}<h_{1} \vee h_{1}<x_{2}<h_{2}\right\}$. 
There is a stationary (basic) flow in $\Pi$ having the representation

$$
\begin{gathered}
\widehat{v}_{1}\left(x_{1}, x_{2}\right)=\left\{\begin{array}{l}
-\frac{g \sin \alpha}{2 \nu_{1}} x_{2}^{2}+a_{1} x_{2} ; \quad 0 \leq x_{2} \leq h_{1}, \\
-\frac{g \sin \alpha}{2 \nu_{2}} x_{2}^{2}+a_{2} x_{2}+b_{2} ; \quad h_{1} \leq x_{2} \leq h_{2},
\end{array} \widehat{v}_{2}\left(x_{1}, x_{2}\right) \equiv 0 .\right. \\
\widehat{p}\left(x_{1}, x_{2}\right)=\left\{\begin{array}{l}
{\left[\varrho_{1} g\left(h_{1}-x_{2}\right)+\varrho_{2} g\left(h_{2}-h_{1}\right)\right] \cos \alpha+p_{a} ;} \\
\varrho_{2} g\left(h_{2}-x_{2}\right) \cos \alpha+p_{a} .
\end{array}\right.
\end{gathered}
$$

The coefficients in these equations are computed as

$$
\begin{gathered}
a_{1}=\frac{g \sin \alpha}{\nu_{2} r}\left[\left(h_{2}-h_{1}\right)+\frac{\varrho_{1}}{\varrho_{2}} h_{1}\right], \quad a_{2}=\frac{g \sin \alpha}{\nu_{2}} h_{2}, \\
b_{2}=\frac{g \sin \alpha h_{1}}{2 r \nu_{2}}\left[\frac{\varrho_{1}}{\varrho_{2}} h_{1}+r h_{1}+2\left(h_{2}-h_{1}\right)-2 r h_{2}\right] .
\end{gathered}
$$

This basic flow is unidirectional and steady-state. The fluxes $F_{j}(j=1,2)$ over an arbitrary cross-section of the flow subdomains $\Pi_{j}(j=1,2)$ which are defined by

$$
F_{1}:=\int_{0}^{h_{1}} v_{1}\left(x_{1}, x_{2}\right) \mathrm{d} x_{2}, \quad F_{2}:=\int_{h_{1}}^{h_{2}} v_{1}\left(x_{1}, x_{2}\right) \mathrm{d} x_{2},
$$

are constant and their positive values can be calculated using the given layer heights $h_{2}>h_{1}>0$. This calculation yields

$$
\begin{gathered}
F_{1}=\frac{1}{6} \frac{g \sin \alpha}{\nu_{2} r} h_{1}^{2}\left[2 \frac{\varrho_{1}}{\varrho_{2}} h_{1}+3\left(h_{2}-h_{1}\right)\right]>0, \\
F_{2}=\frac{1}{6} \frac{g \sin \alpha}{\nu_{2} r}\left(h_{2}-h_{1}\right)\left[2 r\left(h_{2}-h_{1}\right)^{2}+6 h_{1}\left(h_{2}-h_{1}\right)+3 \frac{\varrho_{1}}{\varrho_{2}} h_{1}^{2}\right]>0 .
\end{gathered}
$$

The unknown flow-domain $\Omega(t), t \geq 0$ is then transformed onto the uniform double strip $\Pi$ which is the equilibrium domain. In $\Pi$ the basic solution $\widehat{\mathbf{v}}, \widehat{p}$ is well-known by the above determination.

Now we linearize the original time-dependent Problem (III) over the basic solution in $\Pi$. One derives the following linear problem for the perturbations $\mathbf{u}$ and $q$ of $\widehat{\mathbf{v}}$ and $\widehat{p}$ (resp.). For $\mathbf{x} \in \Pi$ and $t \in(0, T)$ the governing equations are

$$
\left\{\begin{array}{l}
\partial_{t} \mathbf{u}-\nu \varrho \nabla^{2} \mathbf{u}+\nabla q=\mathbf{f} \\
\nabla \cdot \mathbf{u}=0
\end{array}\right.
$$

with the initial condition and the no-slip boundary condition

$$
\left.\mathbf{u}\right|_{t=0}=\mathbf{u}_{0}, \quad(\mathbf{x} \in \Pi),\left.\quad \mathbf{u}\right|_{S}=\mathbf{0}, \quad(t \in(0, T)),
$$

At $x_{2}=h_{1}$ we obtain

$$
\left\{\begin{array}{l}
{\left.[\mathbf{u}]\right|_{x_{2}=h_{1}}=\mathbf{0}, \quad \partial_{t} \psi_{1}-u_{2}=0,\left.\quad\left[\partial_{2} u_{1}+\partial_{1} u_{2}\right]\right|_{x_{2}=h_{1}}=0,} \\
\left.t\left[q-2 \nu \varrho \partial_{2} u_{2}\right]\right|_{x_{2}=h_{1}}-\left(g \psi_{1}-\beta \partial_{1}^{2} \psi_{1}\right)=f_{3},
\end{array}\right.
$$

and at $x_{2}=h_{2}$ one gets

$$
\left\{\begin{array}{l}
\partial_{t} \psi_{2}-u_{2}=0, \quad \partial_{2} u_{1}+\partial_{1} u_{2}=0 \\
q-2 \nu_{2} \varrho_{2} \partial_{2} u_{2}-\left(g \psi_{2}-\beta \partial_{1}^{2} \psi_{2}\right)=f_{4}
\end{array}\right.
$$


In Eqs. (26), (27) the value $\beta$ denotes some constant derived from surface tension. To end this survey let us formulate the main result on Problem (III), namely the following local in time existence result. The detailed proof will be given in a forthcoming paper. It can be realized in a similar manner as in [13].

Theorem 3. Let $3<l<7 / 2, T>0$ be arbitrary and $\alpha>0$ sufficiently small. Then there exists a positive number $\delta$ such that for $\psi_{j}^{(0)}(j=1,2)$ and $\mathbf{u}_{0}$ satisfying appropriate compatibility conditions at $x_{2}=0$ and $x_{2}=h_{j}$ and the estimate

$$
\left\|\psi_{j}^{(0)}\right\|_{W_{2}^{l}(\mathbb{R})}+\left\|\mathbf{u}_{0}\right\|_{W_{2}^{l-1 / 2}(\Pi)}<\delta,
$$

Problem (III) has a solution $\left(\mathbf{u}, q, \psi_{j}\right)$ such that $\psi_{j} \in W_{2}^{l+1 / 2, l / 2+1 / 4}(\mathbb{R} \times(0, T)), \mathbf{u} \in$ $\left[W_{2}^{l, l / 2}(\Pi \times(0, T))\right]^{2}, \nabla q \in W_{2}^{l-2, l / 2-1}(\Pi \times(0, T)),\left.q\right|_{h_{j}} \in W_{2}^{l-3 / 2, l / 2-3 / 4}(\mathbb{R} \times(0, T))$.

\section{References}

[1] F. Abergel and J. L. Bona, A mathematical theory for viscous free-surface flows over a perturbed plane, Arch. Rat. Mech. Anal. 118 (1992), 71-93.

[2] Ch. R. Doering and J. D. Gibbon, Applied Analysis of the Navier-Stokes Equations, Cambridge Texts in Applied Mathematics, Cambridge University Press, 1995.

[3] O. A. Ladyzhenskaya and V. G. Osmolovskii, On the free surface of a fluid layer over a solid sphere, Vestnik Leningrad. Univ. Mat. 13 (1976), no. 3, 25-30 (in Russian); English transl.: Vestnik Leningrad. Univ. Math. 9 (1981), 197-203.

[4] S. A. Nazarov and K. Pileckas, On noncompact free boundary problems for the plane stationary Navier-Stokes equations, J. Reine Angewandte Mathematik 438 (1993), 103141.

[5] K. Pileckas, Solvability of a problem of plane motion of a viscous incompressible fluid with noncompact free boundary, Diff. Equ. Appl. Inst. of Math. Cybern. Acad. Sci. Lit. SSR 30 (1981), 57-96 (in Russian) [cf. also Zap. Nauchn. Sem. LOMI 110 (1981), 174-179 (in Russian); English transl. J. Sov. Math. 25 (1984), 927-931].

[6] K. Pileckas, The example of nonuniqueness of the solutions to a noncompact free boundary problem for the Navier-Stokes system, Diff. Equ. Appl. Inst. of Math. Cybern. Acad. Sci. Lit. SSR 42 (1988), 59-65 (in Russian).

[7] K. Pileckas and J. Socolowsky, Viscous two-fluid flows in perturbed unbounded domains, Math. Nachrichten 278 (2005), 589-623.

[8] V. V. Pukhnachov, Free boundary problems in the theory of thermo-capillary convection, In: A. Bossavit, A. Damlamian and M. Fremond (eds.): Free Boundary Problems: Applications and Theory, Vol. IV, Research Notes in Mathematics 121, Pitman Advanced Publishing program, Boston, 1985, 405-415.

[9] J. Socolowsky, The solvability of a free boundary problem for the stationary Navier-Stokes equations with a dynamic contact line, Nonlinear Analysis, 21 (1993), 763-784.

[10] J. Socolowsky, On nonisothermal two-fluid channel flows, Mathematical Modelling and Analysis 12 (2007), 143-156.

[11] V. A. Solonnikov, Problems with free boundaries and with moving contact points for twodimensional stationary Navier-Stokes equations, Zap. Nauchn. Sem. St.-Peterburg. Otdel. Mat. Inst. Steklova (POMI) 213 (1994), 179-205 (in Russian). 
[12] V. A. Solonnikov, On some free boundary problems for the Navier-Stokes equations with moving contact points and lines, Math. Annalen 302 (1995), 743-772.

[13] Y. Teramoto, On the Navier-Stokes flow down an inclined plane, J. Math. Kyoto Univ. 32 (1992), 593-619.

[14] E. Zadrzyńska, Free boundary value problems for nonstationary Navier-Stokes equations, Diss. Math. 424 (2004). 
